\title{
HPV vaccine gets a shot in the arm
}

L -ate September saw the Michigan Senate approve two bills that will require girls in Michigan entering the sixth grade to be vaccinated against the two strains of the sexually transmitted virus human papillomavirus (HPV) that cause $70 \%$ of all cervical cancers.

The two bills, if approved by the State Assembly, will mean that starting in the 2007-2008 school year, parents in Michigan will be the first in the country required to ensure that their daughters have been vaccinated against HPV before entering sixth grade. But, as with legislation mandating other vaccines (such as those against measles and rubella), the bills do allow parents to opt out of the HPV vaccine requirement for medical, moral, or philosophical reasons. This opt-out clause might be used more often than for other mandatory vaccines, as some critics feel that because HPV is a sexually transmitted disease, providing the vaccination to girls before they become sexually active endorses underage sex and promiscuity. However, State Senator Beverley Hammerstrom (R), who sponsored these bills, told the JCI that "Requiring immunizations for school entry has prov-

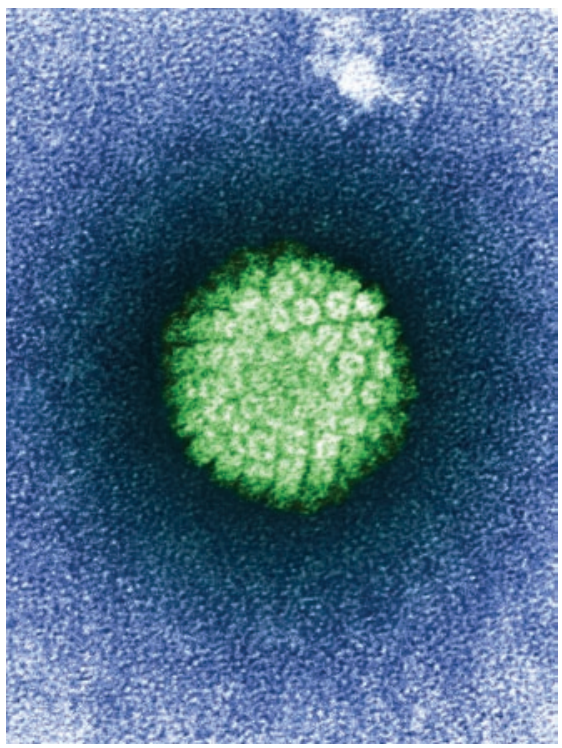

Nearly all cases of cervical cancer are caused by infection with an oncogenic strain of HPV. A new vaccine is now available that protects against infection with HPV16 and HPV18, the two strains of HPV that cause $70 \%$ of cervical cancer. Photo credit: Photo Researchers Inc. en to be the single most important health policy enacted in Michigan to ensure that all children, regardless of economic levels, receive vaccines against diseases. Adding the HPV vaccine, which prevents the virus that can cause cervical cancer, is a natural extension to that list of required vaccines."

Also in late September, the European Medicines Agency (EMEA) approved Gardasil (produced by Merck), the only HPV vaccine currently available in the United States, for use in the 25 European Union (EU) countries. EMEA approved Gardasil for use in children and adolescents aged 9 to 15 years and in adult females aged 16 to 26 years. This followed the June 2006 FDA approval of Gardasil for use in girls and women aged 9 to 26 years, as well as approval by regulatory agencies in Australia, Brazil, Canada, Mexico, and New Zealand. As the JCI was going to press, Gardasil was due to be launched in some EU countries at the end of October and in the remaining countries in early 2007.

Gardasil not only protects against infection with HPV16 and HPV18, the two strains of HPV that account for $70 \%$ of cervical cancer, but also protects against infection with HPV6 and HPV11 - the strains of HPV that cause approximately $90 \%$ of external genital warts. By contrast, the vaccine Cervarix, which is being developed by GlaxoSmithKline, only protects against HPV16 and HPV18. Importantly, the clinical trials of both Cervarix (for which GlaxoSmithKline is awaiting EMEA approval and filing for FDA approval) and Gardasil showed that vaccination provided almost $100 \%$ protection against the development of HPV16- and HPV18-associated precancerous cervical lesions. The generation of such vaccines has therefore been hailed as "excellent news" by Margaret Stanley, an expert in HPV research at the University of Cambridge (1), and as "one of the most important advances in women's health in recent years" by Carolyn Runowicz, president of the American Cancer Society (2).

Optimal protection against infection with HPV requires a course of three doses of Gardasil, with two months between the first and second doses and four months between the second and third doses. The cost is approximately $\$ 120$ and $€ 120$ per dose in the United States and Europe, respectively. It has been suggested that this might be too expensive for developing countries - which have much higher death rates from cervical cancer than do developed countries, largely because they lack organized screening programs - meaning that many of the women in need of this vaccine will not get vaccinated (3). It is also possible that this high cost might mean that some socioeconomic groups in the United States will remain unvaccinated. Several health insurance companies have agreed that vaccination with Gardasil will be covered under preventive medicine health care plans, and the CDC's Advisory Committee on Immunization Practices has recommended that Gardasil be covered by the Vaccines for Children Program (which provides no-cost immunizations to children covered by Medicaid, Alaska Native and American Indian children, and some uninsured and underinsured children). However, some girls do not qualify for the Vaccines for Children Program but come from families too poor to have health insurance or to pay for the vaccine themselves.

In addition to the relatively high cost, there are other drawbacks to HPV vaccines. Although Gardasil protects against infection with HPV6, HPV11, HPV16, and HPV18, it will not prevent individuals already infected with either HPV16 or HPV18 from developing cervical cancer, which can occur up to 10 years after infection. It will also not prevent individuals infected with other oncogenic strains of HPV from developing cancer. Experts believe that for these reasons it is important that cervical cancer screening programs are not abandoned, even when herd immunity to HPV16 and HPV18 begins to increase.

\section{Karen Honey}

1. Sanofi Pasteur MSD. 2006. Gardasil: the vaccine that can prevent cervical cancer approved for use in the European Union. http://www.spmsd.com/ press/Gardasil\%20AMM\%20-\%20english\%20press \%20release\%20accord\%20dif-\%20220906.pdf.

2. American Cancer Society. 2006. HPV vaccine recommended for girls aged 11-12: protects against virus that causes cervical cancer, genital warts. http://www.cancer.org/docroot/NWS/content/ NWS_1_1x_HPV_Vaccine_Recommended_for_ Girls_Aged_11-12.asp.

3. Katz, I.T., and Wright, A.A. 2006. Preventing cervical cancer in the developing world. N. Engl. J. Med. 354:1110. 